\title{
Unique metric segments in the hyperspace over a strictly convex Minkowski space
}

\author{
Agnieszka Bogdewicz • Jerzy Grzybowski
}

Received: 28 February 2012 / Accepted: 26 May 2012 / Published online: 19 July 2012 (C) The Author(s) 2012. This article is published with open access at Springerlink.com

\begin{abstract}
Let $\left(\mathbb{R}^{n},\|\cdot\|_{\mathbb{B}}\right)$ be a Minkowski space (finite dimensional Banach space) with the unit ball $\mathbb{B}$, and let $\varrho_{H}^{\mathbb{B}}$ be the Hausdorff metric induced by $\|\cdot\|_{\mathbb{B}}$ in the hyperspace $\mathcal{K}^{n}$ of convex bodies (compact, convex subsets of $\mathbb{R}^{n}$ with nonempty interior). Schneider (Bull. Soc. Roy. Sci. Li 'ege 50:5-7, 1981) characterized pairs of elements of $\mathcal{K}^{n}$ which can be joined by unique metric segments with respect to $\varrho_{H}$ - the Hausdorff metric induced by the Euclidean norm $\|\cdot\|_{\mathrm{B}^{n}}$. In Bogdewicz and Grzybowski (Banach Center Publ., Warsaw, 75-88, 2009) we proved a counterpart of Schneider's theorem for the hyperspace $\left(\mathcal{K}^{2}, \varrho_{H}^{\mathbb{B}}\right)$ over any two-dimensional Minkowski space. In this paper we characterize pairs of convex bodies in $\mathcal{K}^{n}$ which can be joined by unique metric segments with respect to $\varrho_{H}^{\mathbb{B}}$ for a strictly convex unit ball $\mathbb{B}$ and an arbitrary dimension $n$ (Theorem 3.1).
\end{abstract}

Keywords Convex body $\cdot$ Strict convexity $\cdot$ Minkowski space $\cdot$ Hausdorff metric . Metric segment

Mathematics Subject Classification (2000) Primary 52A10 - 52A29;

Secondary 52A99

\footnotetext{
A. Bogdewicz $(\varangle)$

Faculty of Mathematics and Computer Science,

Warsaw University of Technology, Pl. Politechniki 1, 00-661 Warsaw, Poland

e-mail: A.Bogdewicz@mini.pw.edu.pl; abogde@mini.pw.edu.pl

J. Grzybowski

Faculty of Mathematics and Computer Science,

Adam Mickiewicz University, Umultowska 87, 61-614 Poznan, Poland

e-mail: jgrz@amu.edu.pl
} 


\section{Preliminaries}

Let $\mathcal{K}^{n}$ be the family of convex bodies (nonempty, compact, convex subsets of the space $\mathbb{R}^{n}$ ). The Minkowski addition and multiplication by scalars are defined by the formulae

$$
\begin{aligned}
A_{1}+A_{2} & :=\left\{a_{1}+a_{2} \mid a_{1} \in A_{1}, a_{2} \in A_{2}\right\}, \\
t A & :=\{t a \mid a \in A\},
\end{aligned}
$$

for $A_{1}, A_{2}, A \in \mathcal{K}^{n}$ and $t \in R$.

A Minkowski space is a finite dimensional normed linear space $\left(\mathbb{R}^{n},\|\cdot\|\right.$ ) (see Thompson 1996). Let $\mathbb{B}$ be the unit ball determined by the norm $\|\cdot\|$ :

$$
\mathbb{B}:=\left\{x \in \mathbb{R}^{n} \mid\|x\| \leq 1\right\} .
$$

Then $\mathbb{B}$ is a convex body (i.e., a compact, convex subset of $\mathbb{R}^{n}$ with nonempty interior) symmetric at 0 . Conversely, every convex body $A$ symmetric at 0 determines a norm, $\|\cdot\|_{A}$, usually referred to as the Minkowski functional

$$
\|x\|_{A}:=\inf \{t>0 \mid x \in t A\}
$$

(see Thompson 1996, p.17).

Let $\varrho_{H}^{\mathbb{B}}$ be the Hausdorff metric in $\mathcal{K}^{n}$ associated with the metric $\varrho^{\mathbb{B}}$ induced by the norm $\|\cdot\|_{\mathbb{B}}$ (compare Thompson 1996):

$$
\varrho_{H}^{\mathbb{B}}\left(A_{1}, A_{2}\right):=\max \left\{\inf \left\{\varepsilon>0 \mid A_{1} \subset A_{2}+\varepsilon \mathbb{B}\right\}, \inf \left\{\varepsilon>0 \mid A_{2} \subset A_{1}+\varepsilon \mathbb{B}\right\}\right\}
$$

for every $A_{1}, A_{2} \in \mathcal{K}^{n}$.

The Hausdorff metric induced by the Euclidean norm $\|\cdot\|_{\mathrm{B}^{n}}$ is denoted by $\varrho_{H}$.

Let $(X, \varrho)$ be a metric space. For any $a, b \in X$ a point $c \in X$ such that

$$
\varrho(a, c)=\varrho(c, b)=\frac{1}{2} \varrho(a, b)
$$

is called a metricmidpoint of the pair $(a, b)$. A metricsegment with endpoints $a, b$ is a subset of $X$ isometric to the interval $[0, \varrho(a, b)]$.

Every metric segment with endpoints $a, b$ is a subset of the set

$$
\{x \in X \mid \varrho(a, b)=\varrho(a, x)+\varrho(x, b)\}
$$

which is called $d$-segment in the literature; see, e.g., Boltyanski (1997), Chapter II, and Martini and Swanepoel (2004), paragraph 3.2 (it should be noticed that already Menger in Menger (1928), Part I, used $d$-segments to extend suitably the usual convexity notion to metric convexity; see also Blumenthal $(1953,1970)$, paragraph 14 , and again Martini and Swanepoel (2004), paragraph 3.

The affine segment in $\mathcal{K}^{n}$ with endpoints $K, L \in \mathcal{K}^{n}$ is defined by the formula

$$
\triangle(K, L):=\{(1-t) K+t L \mid t \in[0,1]\} .
$$


The affine midpoint of the pair $(K, L)$ is the set $\frac{1}{2}(K+L)$.

Let us recall that in any normed linear space $X$ every two nonempty convex compact sets $K, L \subset X$ either can be joined by a unique metric segment or there exists an infinite family of metric segments joining $K$ and $L$. A pair $(K, L)$ has a unique metric segment if and only if it has a unique metric midpoint (see Jongmans 1979, p. 244).

For $A \in \mathcal{K}^{n}$ the abbreviations $\operatorname{bd} A, \operatorname{cl} A$, int $A$ and $\operatorname{relint} A$ denote boundary, closure, interior and relative interior of $A, \operatorname{conv} A$ is the convex hull of $A$ and is the affine hull of $A$ (the smallest affine subspace containing $A$ ). For distinct points $a, b \in \mathbb{R}^{n}$ let $\triangle(a, b)$ be the usual segment with endpoints $a, b$. For $A_{1}, A_{2} \in \mathcal{K}^{\mathbb{B}}$

$$
A_{1} \vee A_{2}:=\operatorname{conv}\left(A_{1} \cup A_{2}\right)
$$

Let $A \in \mathbb{R}^{n}$ be a convex set. A face of $A$ is a convex subset $F \subset A$ such that each segment $\triangle(x, y) \subset A$ with $F \cap$ relint $\triangle(x, y) \neq \emptyset$ is contained in $F$ or, equivalently, such that $x, y \in A$ and $(x+y) / 2 \in F$ implies $x, y \in F$. If $\{e\}$ is a face of $A$, then $e$ is called an extreme point of $A$. In other words, $e$ is an extreme point of $A$ if and only if it cannot be written in the form $e=(1-\lambda) x+\lambda y$ with $x, y \in A, x \neq y$ and $\lambda \in(0,1)$ (see Schneider 1993). By $\operatorname{ext} A$ we denote the set of all extreme points of $A$.

Let $\mathbb{R}_{+} \cdot x:=\{t x \mid t \geq 0\}$ for $x \in \mathbb{R}^{n}$. Let now $A \subset \mathbb{R}^{n}$. A tangent cone of $A$ at $a \in \operatorname{bd} A$ is the set

$$
T_{a} A:=\bigcup_{\lambda \geq 0} \lambda(A-a) .
$$

Obviously, if $A$ is strictly convex then $T_{a} A=\operatorname{int} T_{a} A \cup\{a\}$.

It is easy to prove the following:

Proposition 1.1 Let $A \in \mathcal{K}^{n}, a \in \mathrm{bd} A$ and let $\mathbb{B}$ be a convex body symmetric at 0 . Then for every $u \in \mathbb{B}$ the following conditions are equivalent:

(i) $(\mathbb{B}-u) \cap(A-a)=\{0\}$

(ii) $T_{u} \mathbb{B} \cap(A-a)=\{0\}$

(iii) $\left(a+T_{u} \mathbb{B}\right) \cap A=\{a\}$.

Let $A \subset \mathbb{R}^{n}$ and let $H \subset \mathbb{R}^{n}$ be a hyperplane. We say that $H$ supports $A$ at $a$ (denoted by $H(a)$ ) if $a \in A \cap H$ and $A$ is contained in one of the closed halfspaces bounded by $H$. We denote the halfspace containing $A$ by $H^{+}$, the other halfspace by $H^{-}$, and the support set $A \cap H(a)$ by $F(A, a)$.

If $(X,\|\cdot\|)$ is a normed linear space and if $x, y \in X$, then we say that $x$ is normal to $y$ and write $x \dashv y$ if $\|x+\alpha y\| \geq\|x\|$ for all $\alpha \in \mathbb{R}$ (see Thompson 1996, p.78). We note that this normality is also known as Birkhoff orthogonality; see Alonso et al. (2012). Geometrically this means that $x \dashv y$ if and only if the line $x+\mathbb{R} \cdot y$ supports the ball $\|x\| \cdot \mathbb{B}$ at $x$. This implies (by the Hahn-Banach theorem) that the line $x+\mathbb{R} \cdot y$ lies in some hyperplane which supports $\|x\| \cdot \mathbb{B}$ at $x$.

Let $H$ be a hyperplane in $X$ and let $x \in X$. We say that $x$ is normal to $H$ and write $x \dashv H$ if $H$ supports $\|x\| \cdot \mathbb{B}$ at $x$. 
The following theorem is well known.

Theorem 1.2 Let $K, L \in \mathcal{K}^{n}, L \not \subset K$ and $K \not \subset L$. Then there exists a hyperplane $H \subset \mathbb{R}^{n}$ supporting all the sets $(1-t) K+t L$, where $t \in[0,1]$.

Let $K, L \in \mathcal{K}^{n}$. We shall use the following notation:

$$
U_{K, L}:=\{u \in \operatorname{bd} \mathbb{B} \mid h(K, u) \geq h(L, u)\},
$$

where $h(K, u)$ is the value of the support function of the body $K$ at $u$, and

$$
\operatorname{bd}_{K, L} A:=\left\{A(u) \mid u \in U_{K, L}\right\},
$$

where $A(u)$ is a support set (face) of a body $A$. Let us notice that $\operatorname{bd} A=\operatorname{bd}_{K, L} A \cup$ $\mathrm{bd}_{L, K} A$.

Let $K, L \in \mathcal{K}^{n}, K \not \subset L$, and $L \not \subset K$. We define $K * L$ by

$$
K * L:=\bigcap\left\{H^{+}(K \cup L) \mid H \text { supports } K \text { and } L\right\} \text {. }
$$

Let $x \in \mathbb{R}^{n}$. We say that the set $K$ is visible from $x$ with respect to $L$ if there exists $k \in K$ such that $L \cap \operatorname{relint}(k \vee x)=\emptyset$.

Here are two elementary propositions.

Proposition 1.3 Let $C \in \mathcal{K}^{n}$ and $U \subset$ bdB. If int $C \neq \emptyset$ and $\operatorname{bd} C=\bigcup_{u \in U} C(u)$, then

$$
C=\bigcap_{u \in U}\{x \mid\langle x, u\rangle \leq h(C, u)\} .
$$

Proposition 1.4 Assume $A, C \in \mathcal{K}^{n}$ and $A$ to be a summand of $C$. Let $U \subset$ bdBB. If $C=\bigcap_{u \in U}\{x \mid\langle x, u\rangle \leq h(C, u)\}$, then $A=\bigcap_{u \in U}\{x \mid\langle x, u\rangle \leq h(A, u)\}$.

Proof Let $A, B, C \in \mathcal{K}^{n}$ and $C=A+B$. Then

$$
\begin{aligned}
A+B & \subset \bigcap_{u \in U}\{x \mid\langle x, u\rangle \leq h(A, u)\}+\bigcap_{u \in U}\{x \mid\langle x, u\rangle \leq h(B, u)\} \\
& \subset \bigcap_{u \in U}\{x \mid\langle x, u\rangle \leq h(C, u)\}=C=A+B .
\end{aligned}
$$

The paper is organized as follows:

In Sect. 2 we recall the Schneider theorem (Theorem 2.1) which deals with the hyperspace $\left(\mathcal{K}^{n}, \rho_{H}\right)$ and our partial generalization for $n=2$ of this theorem over the hyperspace $\left(\mathcal{K}^{2}, \rho_{H}^{\mathbb{B}}\right)$ for any two-dimensional Minkowski ball $\mathbb{B}$ (Theorem 2.2). In Sect. 3, finally, we present our new result (Theorem 3.1).

Sections 4 and 5 contain the proof of Theorem 3.1. In Sect. 6 we show how to construct examples. Section 7 contains final remarks. 


\section{Survey of results}

Schneider (1981) characterized pairs of elements of $\mathcal{K}^{n}$ which can be joined by unique metric segments with respect to $\varrho_{H}$.

Theorem 2.1 (Schneider 1981) Let $K, L \in \mathcal{K}^{n}$. Then the following conditions are equivalent:

(i) the sets $K$ and L have a unique metric segment joining them, with respect to the metric $\varrho_{H}$;

(ii) either

(a) $K=L+\lambda B^{n}$ or $L=K+\lambda B^{n}$ for some $\lambda>0$ or else,

(b) $\operatorname{dim} L<n$ and $K=L+u$ for some $u \in \mathbb{R}^{n}$ orthogonal to.

In our paper Bogdewicz and Grzybowski (2009) we extended Schneider's theorem to the hyperspace $\left(\mathcal{K}^{2}, \varrho_{H}^{\mathbb{B}}\right)$ over any two-dimensional Minkowski space:

Theorem 2.2 (Bogdewicz and Grzybowski (2009), Theorem 4.9) Let $K, L \in \mathcal{K}^{n}$ for $n=2$. Then the following conditions are equivalent:

(i) the sets $K$ and L have a unique metric segment joining them, with respect to the metric $\varrho_{H}^{\mathbb{B}}$;

(ii) $K=L+\lambda F$ or $L=K+\lambda F$ for some $\lambda>0$ and a face $F$ of the unit ball $\mathbb{B}$; moreover, the sets $L$ and $F$ (or $K$ and $F$, respectively) satisfy one of the conditions 1-4 from the Table of conditions (see Bogdewicz and Grzybowski 2009, p. 3).

The conditions from part (ii) of Theorem 2.2 are rather complicated. In Theorem 3.1 we show that these conditions can be reduced under the extra assumption that the unit ball $\mathbb{B}$ is strictly convex. Moreover, we extend Theorem 2.2 to arbitrary dimension $n$.

\section{Main theorem}

In this section we characterize pairs of convex bodies in $\mathcal{K}^{n}$ which can be joined by unique metric segments with respect to $\varrho_{H}^{\mathbb{B}}$ for a strictly convex unit ball $\mathbb{B}$ and an arbitrary dimension $n$.

Theorem 3.1 Let $\mathbb{B}$ be strictly convex and let $K, L \in \mathcal{K}^{n}$. Then the following conditions are equivalent:

(i) the sets $K$ and L have a unique metric segment joining them, with respect to the metric $\varrho_{H}^{\mathbb{B}}$;

(ii) either

(a) $K=L+\lambda \mathbb{B}$ or $L=K+\lambda \mathbb{B}$ for some $\lambda>0$

or else,

(b) $K=L+t u$ for $t>0$ and for some $u \in$ bdB such that:

$\left(T_{u} \mathbb{B}+l\right) \cap L=\{l\}$ or $\left(T_{-u} \mathbb{B}+l\right) \cap L=\{l\}$ for every $l \in \mathrm{bd} L$. 
The proof of Theorem 3.1 is divided into two parts. The implication (ii) $\Rightarrow$ (i) is proved in Sect. 4 (Theorem 4.5). The converse implication is proved in Sect. 5 (Theorem 5.7).

Notice that if $\mathbb{B}$ is assumed to be the Euclidean ball, then Theorem 3.1 is equivalent to Schneider's Theorem 2.1.

In Minkowski spaces, the Euclidean notion of orthogonality is replaced by the notion of normality (see Thompson 1996, p.75). Let us notice that if $\operatorname{dim} L<n$, then condition (ii) (b) in Theorem 3.1 is equivalent to condition (ii) (b) in Theorem 2.1 (with orthogonality replaced by normality). Since in Theorem 3.1 we do not assume that $\operatorname{dim} L<n$, it follows that Theorem 3.1 is an essential extension of Schneider's Theorem 2.1.

It is worth mentioning that for the unit ball $\mathbb{B}$ being strictly convex and smooth we obtain the following counterpart of Schneider's result:

Corollary 3.2 Let $\mathbb{B}$ be strictly convex and smooth, and let $K, L \in \mathcal{K}^{n}$. Then the following conditions are equivalent:

(i) the sets $K$ and L have a unique metric segment joining them, with respect to the metric $\varrho_{H}^{\mathbb{B}}$;

(ii) either

(a) $K=L+\lambda \mathbb{B}$ or $L=K+\lambda \mathbb{B}$ for some $\lambda>0$

or else,

(b) $\operatorname{dim} L<n$ and $K=L+u$ for some $\frac{u}{\|u\|} \in \operatorname{bdB}$ such that $u \dashv \operatorname{aff} L$.

Proof Assume that the unit ball $\mathbb{B}$ is strictly convex and smooth. Then for every $u \in \mathbb{B}$ there exists a unique hyperplane $H(u)$ supporting $\mathbb{B}$ at $u$, i.e., $u \dashv H(u)$ and $H \cap \mathbb{B}=\{u\}$. In this case the tangent cone $T_{u} \mathbb{B}$ is, in fact, the whole halfspace $H^{-}(u)$. Then, condition (ii) (b) in Theorem 3.1 implies $\operatorname{dim} L<n$ and $u \dashv \operatorname{aff} L$.

\section{Proof of Theorem 3.1. Sufficiency of (ii)}

In this section we prove that for every pair of elements of $\mathcal{K}^{n}$ described in Theorem 3.1 (ii) there is a unique metric segment, with respect to $\varrho_{H}^{\mathbb{B}}$, joining the elements of this pair.

The following lemma does not require the assumption on the unit ball $\mathbb{B}$ to be strictly convex.

Lemma 4.1 Let $L \in \mathcal{K}^{n}$ and let $K=L+\lambda \mathbb{B}$ for some $\lambda>0$ and a convex body $\mathbb{B}$ symmetric at 0 . Then the sets $K$ and L have a unique metric segment joining them with respect to the metric $\varrho_{H}^{\mathbb{B}}$.

Proof Without loss of generality we may assume that $\varrho_{H}^{\mathbb{B}}(K, L)=\lambda=2$. Then $\frac{1}{2}(K+L)=L+\mathbb{B}$ and

$$
(K+\mathbb{B}) \cap(L+\mathbb{B})=(L+3 \mathbb{B}) \cap(L+\mathbb{B})=L+\mathbb{B} .
$$

Now it suffices to prove that there is no other metric midpoint $S$ of the pair $K, L$ such that $S \subsetneq L+\mathbb{B}$ (compare Bogdewicz 2000). 
Assume that $S \in \mathcal{K}^{n}$ is a metric midpoint of the pair $K, L$. Then $L+2 \mathbb{B} \subset S+\mathbb{B}$. By order law of cancellation $L+\mathbb{B} \subset S$.

In order to prove Lemma 4.4 we need two following simple propositions.

Proposition 4.2 Let $C$ be a convex cone in a vector space $X$, and $A$ be any subset of $X$. Then the following conditions are equivalent:

(i) For any point $x$ not belonging to A at least one of the intersections $A \cap(x+C)$, $A \cap(x-C)$ is empty.

(ii) For any $x, y \in A$ with $y-x \in C$, then $(y-C) \cap(x+C) \subset A$.

Proposition 4.3 Let $C$ be a convex cone in $\mathbb{R}^{n}$ such that $C \backslash\{0\}$ is an open set and $A$ be a closed convex subset of $\mathbb{R}^{n}$. Then the following conditions are equivalent:

(i) For any point $x$ not belonging to $A$ at least one of the intersections $A \cap(x+C)$, $A \cap(x-C)$ is empty.

(ii) For any point $x$ belonging to bd $A$ at least one of the intersections $A \cap(x+C)$, $A \cap(x-C)$ is a singleton $\{x\}$.

Proof Assume (i). Let $x \in$ bd $A$, and let $x_{n}$ tend to $x$, where $x_{n} \notin A$. We can assume that $A \cap\left(x_{n}+C\right)$ is empty. If $y \in x+C, y \neq x$, then $y-x_{n}$ tends to $y-x \in C$. Hence for sufficiently great $n$ we have $y-x_{n} \in C$. Therefore, $y \in x_{n}+C$ and $y \notin A$.

Assume that (i) does not hold. Then for some $x \notin A$ there exist $y, z \in A$ such that $y-x, x-z \in C$. Since $A$ is convex, $\frac{y+z}{2} \in A$ and there exists $w \in \operatorname{bd} A \cap \Delta\left(x, \frac{y+z}{2}\right)$. We have $w=t \frac{y+z}{2}+(1-t) x$ for some $t \in(0,1]$. Then $y-w=\left(1-\frac{t}{2}\right)(y-x)+\frac{t}{2}(x-z) \in$ $C$ and $w-x=\frac{t}{2}(y-x)+\left(1-\frac{t}{2}\right)(x-z) \in C$. Therefore, (ii) does not hold.

Lemma 4.4 Assume that $\mathbb{B}$ is strictly convex. Let $L \in \mathcal{K}^{n}$ and let $K=L+t u$ for $t>0$ and $u \in \mathrm{bdB}$ be such that

$$
\left(T_{u} \mathbb{B}+l\right) \cap L=\{l\} \text { or }\left(T_{-u} \mathbb{B}+l\right) \cap L=\{l\}
$$

for every $l \in \mathrm{bd} L$. Then $K$ and $L$ have a unique metric segment joining them, with respect to the metric $\varrho_{H}^{\mathbb{B}}$.

Proof Without a loss of generality we may assume that $t=2$. First we shall show that $(K+\mathbb{B}) \cap(L+\mathbb{B})=L+u$. Obviously, $L+u \subset(K+\mathbb{B}) \cap(L+\mathbb{B})$. Suppose that $x \in(K+\mathbb{B}) \cap(L+\mathbb{B})$. Then $x=l_{1}+2 u+b_{1}=l_{2}+b_{2}$ for some $l_{1}, l_{2} \in L, b_{1}, b_{2} \in \mathbb{B}$. Hence $x-u=l_{2}+b_{2}-u \in l_{2}+T_{u} \mathbb{B}$ and $x-u=l_{1}+u+b_{1} \in l_{1}-T_{u} \mathbb{B}$. By Propositions 4.2 and 4.3, $x-u \in L$. Therefore, $x \in L+u$.

Now it suffices to prove that there is no other metric midpoint $Y$ of the pair $(K, L)$ such that $Y \subsetneq L+\mathbb{B}$. Let us assume that $Y$ is a metric midpoint of the pair $(K, L)$ such that $Y \subsetneq L+\mathbb{B}$. Then there exists $x \in(\operatorname{bd}(L+u)) \backslash Y$. By assumption $(x-u+$ $\left.T_{u} \mathbb{B}\right) \cap L=\{x-u\}$ or $\left(x-u-T_{u} \mathbb{B}\right) \cap L=\{x-u\}$. Hence 


$$
(x-u+\mathbb{B}) \cap Y \subset(x+\mathbb{B}-u) \cap(L+u) \subset\left(x+T_{u} \mathbb{B}\right) \cap(L+u)=\{x\}
$$

or

$$
(x+u+\mathbb{B}) \cap Y \subset(x-(\mathbb{B}-u)) \cap(L+u) \subset\left(x-T_{u} \mathbb{B}\right) \cap(L+u)=\{x\} .
$$

Since $x \notin Y$, in the first case $x-u \notin Y+\mathbb{B}$ and $\varrho_{H}^{\mathbb{B}}(Y, L)>1$, and in the second case $x+u \notin Y+\mathbb{B}$ and $\varrho_{H}^{\mathbb{B}}(Y, K)>1$, a contradiction.

The following theorem is a consequence of Lemmas 4.1 and 4.4.

Theorem 4.5 Let $B$ be strictly convex and let $K, L \in \mathcal{K}^{n}$. If either

(a) $K=L+\lambda \mathbb{B}$ or $L=K+\lambda \mathbb{B}$ for some $\lambda>0$ or else,

(b) $K=L+t u$ for some $u \in \mathrm{bd} \mathbb{B}$ and $t>0$ such that $\left(T_{u} \mathbb{B}+l\right) \cap L=\{l\}$ or $\left(T_{-u} \mathbb{B}+l\right) \cap L=\{l\}$ for every $l \in \mathrm{bd} L$ then the sets $K$ and $L$ have a unique metric segment joining them, with respect to the metric $\varrho_{H}^{\mathbb{B}}$.

\section{Proof of Theorem 3.1. Necessity of (ii)}

We are now going to prove that every pair of elements of $\mathcal{K}^{n}$ with a unique metric (with respect to $\varrho_{H}^{\mathbb{B}}$ ) segment joining them is of one of the two types described in condition (ii) in Theorem 3.1. We shall need the following lemmas:

Lemma 5.1 Assume that $\mathbb{B}$ is strictly convex. Let $K, L \in \mathcal{K}^{n}$ have a unique metric midpoint $C \in \mathcal{K}^{n}$ with respect to the metric $\varrho_{H}^{\mathbb{B}}$ and let $\varrho_{H}^{\mathbb{B}}(K, L)=2$. Then for any $c \in \operatorname{ext} C$ there exists $l_{0} \in L$ such that $\left(l_{0}+\mathbb{B}\right) \cap C=\{c\}$ or there exists $k_{0} \in K$ such that $\left(k_{0}+\mathbb{B}\right) \cap C=\{c\}$. Moreover, if $\left(l_{0}+\mathbb{B}\right) \cap C=\{c\}$, then $c \in \operatorname{bd}_{L, K} C$, and if $\left(k_{0}+\mathbb{B}\right) \cap C=\{c\}$, then $c \in \mathrm{bd}_{K, L} C$.

Proof Let $c \in \operatorname{ext} C$. For a sequence $\left\{E_{i}^{+}\right\}_{i \in \mathbb{N}}$ of closed halfspaces containing $c$ let $C_{i}^{\prime}:=C \cap E_{i}^{+}$and $C_{i}:=C \backslash \operatorname{int} E_{i}^{+}$. We can choose a sequence $\left\{E_{i}^{+}\right\}_{i \in \mathbb{N}}$ of closed halfspaces and we can assume that $\operatorname{diam} C_{i}^{\prime} \rightarrow 0$ and $c \in \bigcap_{i \in \mathbb{N}} \operatorname{int} E_{i}^{+}$.

By the assumption, no convex set $C_{i}$ is a metric midpoint of the pair $(K, L)$. Hence $\varrho_{H}^{\mathbb{B}}\left(C_{i}, K\right)>1$ or $\varrho_{H}^{\mathbb{B}}\left(C_{i}, L\right)>1$. Since $C_{i} \subset C \subset(K+\mathbb{B}) \cap(L+\mathbb{B})$, we have $K \cup L \not \subset C_{i}+\mathbb{B}$.

Let $\left(x_{i}\right)_{i \in \mathbb{N}} \subset K \cup L$ be such a sequence that $x_{i} \notin C_{i}+\mathbb{B}$. We can assume that the sequence $\left(x_{i}\right)_{i \in \mathbb{N}}$ converges to a point $x \in K \cup L$. Notice that $\operatorname{dist}(x, C) \geq$ $\operatorname{dist}\left(x_{i}, C_{i}\right)-\left\|x-x_{i}\right\|_{\mathbb{B}}-\varrho_{H}^{\mathbb{B}}\left(C, C_{i}\right) \geq \operatorname{dist}\left(x_{i}, C_{i}\right)-\left\|x-x_{i}\right\|_{\mathbb{B}}-\operatorname{diam} C_{i}^{\prime}$. Since for all $i$ we have $\operatorname{dist}\left(x_{i}, C_{i}\right)>1$, also $\operatorname{dist}(x, C)=1$. Notice also that $\|x-c\|_{\mathbb{B}} \leq$ $\left\|x-x_{i}\right\|_{\mathbb{B}}+\operatorname{dist}\left(x_{i}, C_{i}^{\prime}\right)+\operatorname{diam} C_{i}^{\prime}$. Since for all $i$ we have $\operatorname{dist}\left(x_{i}, C_{i}^{\prime}\right) \leq 1$, also $\|x-c\|_{\mathbb{B}}=1$. Since $\mathbb{B}$ is strictly convex, it follows that $(x+\mathbb{B}) \cap C=\{c\}$. If $x \in L$ we put $l_{0}=x$, and if $x \in K$ we put $k_{0}=x$.

The following lemma is a natural consequence of Lemma 5.1.

Lemma 5.2 Assume $\mathbb{B}$ to be strictly convex. Let $K, L \in \mathcal{K}^{n}$ have a unique metric midpoint $C \in \mathcal{K}^{n}$ with respect to the metric $\varrho_{H}^{\mathbb{B}}$ and let $\varrho_{H}^{\mathbb{B}}(K, L)=2$. For every $c \in \operatorname{ext} C$ there exist unique $k \in K$ and $l \in L$ such that $c=\frac{k+l}{2}$ and 


$$
(k+\mathbb{B}) \cap C=\{c\} \text { and }(k+2 \mathbb{B}) \cap L=\{l\}
$$

or

$$
(l+\mathbb{B}) \cap C=\{c\} \text { and }(l+2 \mathbb{B}) \cap K=\{k\} .
$$

Moreover, if 5.1 holds, then $k \in \operatorname{bd}_{K, L} K, c \in \operatorname{bd}_{K, L} C$, and $l \in \operatorname{bd}_{K, L} L$; if 5.2 holds, then $k \in \operatorname{bd}_{L, K} K, c \in \mathrm{bd}_{L, K} C$, and $l \in \mathrm{bd}_{L, K} L$.

Proof Let $c \in \operatorname{ext} C$. By Lemma 5.1, we can first assume that there exists $l_{0} \in L$ such that $\left(l_{0}+\mathbb{B}\right) \cap C=\{c\}$. The Separation Theorem implies that there exists a hyperplane $H$ such that $\left(l_{0}+\mathbb{B}\right) \subset H^{+}$and $C \subset H^{-}$. Then $c \in H$ and, since $\mathbb{B}$ is strictly convex, it follows that $\left(l_{0}+\mathbb{B}\right) \cap H^{-}=\{c\}$. Then $\operatorname{dist}\left(H, H+l_{0}-c\right)=1$ and $L \subset H^{-}+l_{0}-c$. From the equality $C=\frac{1}{2}(K+L)$ we conclude that $K \subset H^{-}+c-l_{0}$. Then $\left(l_{0}+2 \mathbb{B}\right) \subset H^{+}+c-l_{0}$ and $\left(l_{0}+2 \mathbb{B}\right) \cap K=\left\{2 c-l_{0}\right\}$.

By the assumptions (the ball $\mathbb{B}$ is strictly convex, the point $c$ is an extreme point of $C$ and $C=\frac{K+L}{2}$ ), there exist unique points $k \in K$ and $l \in L$ such that $c=\frac{k+l}{2}$. We put $l=l_{0}$ and $k=2 c-l_{0}$, and in this way we obtain 5.2.

Otherwise, by Lemma 5.1, there exists $k_{0} \in K$ such that $\left(k_{0}+\mathbb{B}\right) \cap C=\{c\}$. In the same manner we obtain 5.2.

As it is well known (compare Jongmans 1979) that if $K \not \subset L$ and $L \not \subset K$, then there exists a hyperplane $H$ supporting $K, L$, and $\frac{1}{2}(K+L)$.

Lemma 5.3 Assume that $\mathbb{B}$ is strictly convex. Let $K, L \in \mathcal{K}^{n}$ have a unique metric midpoint $C \in \mathcal{K}^{n}$ with respect to the metric $\varrho_{H}^{\mathbb{B}}$, and let $\varrho_{H}^{\mathbb{B}}(K, L)=2$. If $K \not \subset L \not \subset K$ and parallel hyperplanes $H_{K}, H_{L}, H_{C}$ support $K, L$, and $C$, respectively, then there exists $u \in \mathrm{bdB}$ such that $H_{K} \cap K=H_{C} \cap C+u=H_{L} \cap L+2 u$.

Moreover, if $H_{C} \cap C \subset \mathrm{bd}_{K, L} C$, then for all $c \in H_{C} \cap C$ we have $(c+u+\mathbb{B}) \cap C=\{c\}$.

Proof Let $c_{1}, c_{2} \in \operatorname{ext}\left(H_{C} \cap C\right) \subset \operatorname{ext} C, c_{1} \neq c_{2}$. There exist unique $l_{i} \in L$ and $k_{i} \in K$ such that $c_{i}=\frac{k_{i}+l_{i}}{2}$ for $i=1$, 2. Lemma 5.2 implies that $\left\|k_{i}-c_{i}\right\|_{\mathbb{B}}=1$ for $i=1,2$. If $k_{1}-c_{1} \neq k_{2}-c_{2}$, then by the strict convexity of $\mathbb{B}$

$$
\left\|\frac{k_{1}+k_{2}}{2}-\frac{c_{1}+c_{2}}{2}\right\|_{\mathbb{B}}=1-\varepsilon<1
$$

for some $\varepsilon>0$. Hence

$$
\frac{c_{1}+c_{2}}{2} \in(K+(1-\varepsilon) \mathbb{B}) \cap(L+(1-\varepsilon) \mathbb{B}) \subset \operatorname{int} C .
$$

But $\frac{c_{1}+c_{2}}{2} \in \operatorname{bd} C$. Therefore, we have $k_{1}-c_{1}=k_{2}-c_{2}$. Put $u:=k_{1}-c_{1}$. Then $\operatorname{ext}\left(H_{K} \cap K\right)=u+\operatorname{ext}\left(H_{C} \cap C\right)=2 u+\operatorname{ext}\left(H_{L} \cap L\right)$, which completes the proof.

Let us mention an important consequence of Lemma 5.3.

Corollary 5.4 Assume that $\mathbb{B}$ is strictly convex. Let $K, L \in \mathcal{K}^{n}$ have a unique metric midpoint $C \in \mathcal{K}^{n}$ with respect to the metric $\varrho_{H}^{\mathbb{B}}$ and let $\varrho_{H}^{\mathbb{B}}(K, L)=2$. If $K \not \subset L \not \subset K$ and int $C=\emptyset$, then there exists $u \in \operatorname{bd} \mathbb{B}$ such that $K=C+u=L+2 u$. 
Moreover, $C=\operatorname{bd}_{K, L} C=\operatorname{bd}_{L, K} C, K=\operatorname{bd}_{K, L} K=\operatorname{bd}_{L, K} K$ and $L=\operatorname{bd}_{K, L} L=$ $\operatorname{bd}_{L, K} L$.

Lemma 5.5 Assume that $\mathbb{B}$ is strictly convex. Let $K, L \in \mathcal{K}^{n}$ have a unique metric midpoint $C \in \mathcal{K}^{n}$ with respect to the metric $\varrho_{H}^{\mathbb{B}}$. If $K \not \subset L \not \subset K$, then

$\operatorname{bd}(K * L) \subset \bigcup\{H \cap C+\mathbb{R} \cdot u \mid H$ supports $K, L$ and $H \cap C+u=H \cap K\}$.

Proof Let $K \not \subset L \not \subset K$. By Lemma 5.3, for every $H$ supporting $K$ and $L$ the intersections $H \cap K, H \cap L$, and $H \cap C$ are pairwise disjoint and are mutual translates. For every $x \in \mathbb{R}^{n}$ the following are equivalent:

(i) $x \notin K * L$,

(ii) $x \notin \operatorname{conv}(K \cup L)$ and each of $K$ and $L$ is visible from $x$ with respect to the other one.

If $x \in H$ and $x \notin H \cap C+\mathbb{R} \cdot u$, where $u \in \mathbb{R}^{n}$ is such that $H \cap K=H \cap C+u$, then each of $K$ and $L$ is visible from $x$ with respect to the other one and $x \notin K \cup L$. Therefore $(K * L) \cap H \subset H \cap C+\mathbb{R} \cdot u$.

Lemma 5.6 Assume that $\mathbb{B}$ is strictly convex. Let $K, L \in \mathcal{K}^{n}$ have a unique metric midpoint $C \in \mathcal{K}^{n}$ with respect to the metric $\varrho_{H}^{\mathbb{B}}$, and let $\varrho_{H}^{\mathbb{B}}(K, L)=2$. If $K \not \subset L \not \subset K$, and $\operatorname{int} C \neq \emptyset$, then

(i) for every $k \in \operatorname{bd}_{K, L} K$ there exists a unique $c \in C$ such that $(k+\mathbb{B}) \cap C=\{c\}$,

(ii) $\operatorname{bd}_{K, L} C$ is not contained in $\operatorname{bd}_{L, K} C$, and for every $c \in \operatorname{bd}_{K, L} C \backslash \mathrm{bd}_{L, K} C$ there exists $k \in \operatorname{bd}_{K, L} K$ such that $c+\mathbb{R}_{+} \cdot(k-c) \subset K * L$. The point $c$ is a unique point for $k$ from (i).

Proof (i) Let $k \in \operatorname{bd}_{K, L} K$ and let $H$ be a hyperplane supporting $K$ at $k$. There exist hyperplanes $H_{C}$ and $H_{L}$ parallel to $H$ supporting, respectively, $C$ and $L$ from the same side. Since $C=\frac{1}{2}(K+L)$, we have $H_{C} \cap C=\frac{1}{2}\left(H \cap K+H_{L} \cap L\right)$. Let $c_{1}, c_{2} \in \operatorname{ext}\left(H_{C} \cap C\right)$ and $c_{1} \neq c_{2}$. There exist unique $k_{1}, k_{2} \in H \cap K$ and $l_{1}, l_{2} \in H_{L} \cap L$ such that $c_{1}=\frac{1}{2}\left(k_{1}+l_{1}\right)$ and $c_{2}=\frac{1}{2}\left(k_{2}+l_{2}\right)$. By Lemma 5.2, we have $\varrho^{\mathbb{B}}\left(k_{1}, c_{1}\right)=1$ and $\varrho^{\mathbb{B}}\left(k_{2}, c_{2}\right)=1$. If $k_{1}-c_{1} \neq k_{2}-c_{2}$ then, by the strict convexity of $\mathbb{B}$, the point $\frac{1}{2}\left(c_{1}+c_{2}\right)$ belongs to the interior of the set $\left(\frac{1}{2}\left(k_{1}+k_{2}\right)+\mathbb{B}\right) \cap\left(\frac{1}{2}\left(l_{1}+l_{2}\right)+\mathbb{B}\right) \subset C$. Therefore $\frac{1}{2}\left(c_{1}+c_{2}\right)$ does not belong to bdC-a contradiction. Thus, $k_{1}-c_{1}=k_{2}-c_{2}$. Put $u=k_{1}-c_{1}$. Then $H \cap K=H_{C} \cap C+u$, and for $c=k-u$ we have $(k+\mathbb{B}) \cap C=\{c\}$

(ii) First we prove that bd ${ }_{K, L} C$ is not contained in $\mathrm{bd}_{L, K} C$. Assume that bd ${ }_{K, L} C \subset$ $\operatorname{bd}_{L, K} C$. Then $\operatorname{bd} C=\bigcup\left\{C(u) \mid u \in \mathcal{U}_{L, K}\right\}$. By Proposition 1.3, $C=$ $\bigcap_{u \in U_{L, K}}\{x \mid\langle x, u\rangle \leq h(C, u)\}$. Let us recall that $C=\frac{K+L}{2}$. By Proposition 1.4 it follows that: $K=\bigcap_{u \in U_{L, K}}\{x \mid\langle x, u\rangle \leq h(K, u)\}$ and $L=$ $\bigcap_{u \in U_{L, K}}\{x \mid\langle x, u\rangle \leq h(L, u)\}$. From the definition of $U_{L, K}$ we conclude that $K \subset L$, which contradicts the assumption.

Let $c \in \operatorname{bd}_{K, L} C \backslash \mathrm{bd}_{L, K} C$. By Lemma 5.3, there exists $u \in \operatorname{bd} \mathbb{B}$ such that $c+u \in$ $\operatorname{bd}_{K, L} K$ and $(k+\mathbb{B}) \cap C=\{c\}$ for $k=c+u$. 
Suppose that $c+\mathbb{R}_{+} \cdot(k-c) \not \subset(K * L)$. There exists a maximal $t \geq 1$ such that $x:=c+t(k-c) \in K * L$. Hence $x \in \operatorname{bd}(K * L)$. By the definition of $K * L$ there exists a hyperplane $H$ nonparallel to $k-c$ and supporting both $K$ and $L$ such that $x \in H$. By Lemma 5.5, also, there exists $u_{1} \in$ bdB such that $(H \cap C)+u_{1}=H \cap K$. Then $x=c_{1}+s u_{1}$ for some $c_{1} \in H \cap C$ and $s \geq 1$.

Hence, by Lemma 5.3, $(x+s \mathbb{B}) \cap C=\left\{c_{1}\right\}$, while $(x+t \mathbb{B}) \cap C=\{c\}$. Then $s=t, c_{1}=c$, and $c \in H \cap C \subset \operatorname{bd}_{K, L} C \cap \mathrm{bd}_{L, K} C-$ a contradiction.

Lemma 5.7 Assume that $\mathbb{B}$ is strictly convex. Let $K, L \in \mathcal{K}^{n}$ have a unique metric midpoint $C \in \mathcal{K}^{n}$ with respect to the metric $\varrho_{H}^{\mathbb{B}}$, and let $\varrho_{H}^{\mathbb{B}}(K, L)=2$. If $K \not \subset L \not \subset K$ and $\operatorname{int} C \neq \emptyset$, then there exists a unique $u \in \operatorname{bd} \mathbb{B}$ such that

$$
\operatorname{bd}(K * L)=\bigcup\{(H \cap C)+\mathbb{R} \cdot u \mid H \text { supports } K \text { and } L\},
$$

where $\left(\operatorname{bd}_{K, L} C \backslash \mathrm{bd}_{L, K} C\right)+u \subset \mathrm{bd} K$ and $\left(\operatorname{bd}_{L, K} C \backslash \mathrm{bd}_{K, L} C\right)+u \subset \operatorname{bd} K$.

Proof By Lemma 5.6, there exists $k \in \operatorname{bd}_{K, L} K$ and there exists $c_{1} \in C$ such that $c_{1}+\mathbb{R}_{+} \cdot\left(k-c_{1}\right) \subset K * L$. Analogously, there exists $l \in \operatorname{bd}_{L, K} L$ and there exists $c_{2} \in C$ such that $c_{2}+\mathbb{R}_{+} \cdot\left(l-c_{2}\right) \subset K * L$.

Denote $u_{1}:=k-c_{1}$ and $u_{2}:=l-c_{2}$. Suppose that $u_{1} \neq-u_{2}$. Then for sufficiently large $t>0$ the segment $S:=\left(c_{1}+t\left(k-c_{1}\right)\right) \vee\left(c_{2}+t\left(l-c_{2}\right)\right)$ and the set $K \vee L$ are disjoint. It follows that there exists $z \in S$ such that both $K$ and $L$ are visible from $z$ with respect to the other. Hence $z \notin K * L$, but $K * L$ is convex. From this contradiction it follows that $u_{1}=-u_{2}$. Hence the set $K * L$ contains a straight line parallel to $u=u_{1}$.

By Lemma 5.6 in our considerations we can take any $c_{1} \in \operatorname{bd}_{K, L} C \backslash \mathrm{bd}_{L, K} C$ and any $c_{2} \in \operatorname{bd}_{L, K} C \backslash \mathrm{bd}_{K, L} C$. Then $\left(\operatorname{bd}_{K, L} C \backslash \mathrm{bd}_{L, K} C\right)+u \subset \operatorname{bd}_{K, L} K$. Obviously, also $\left(\operatorname{bd}_{K, L} C \backslash \operatorname{bd}_{L, K} C\right)-u \subset \operatorname{bd}_{K, L} L$. On the other hand $\left(\operatorname{bd}_{L, K} C \backslash \operatorname{bd}_{K, L} C\right)-u \subset$ $\operatorname{bd}_{L, K} L$ and $\left(\operatorname{bd}_{L, K} C \backslash \operatorname{bd}_{K, L} C\right)+u \subset \operatorname{bd}_{L, K} K$.

Let $H$ be a hyperplane supporting $K$ and $L$. By Lemma 5.3 there exists $v \in$ bdB such that $H \cap C+v=H \cap K$. Notice that $H \cap(K * L) \subset H \cap C+\mathbb{R} \cdot v$. On the other hand, since $K * L$ contains a straight line parallel to $u$, the hyperplane $H$ is parallel to $u$ and $H \cap C+\mathbb{R} \cdot u \subset H \cap \operatorname{bd}(K * L)$. Therefore, $u$ and $v$ are parallel, which proves the lemma.

Theorem 5.8 Let $\mathbb{B}$ be strictly convex and let $K, L \in \mathcal{K}^{n}$. If the sets $K$ and $L$ have the unique metric segment joining them with respect to the metric $\varrho_{H}^{\mathbb{B}}$, then either

(a) there exists $\lambda>0$ such that $K=L+\lambda \mathbb{B}$ or $L=K+\lambda \mathbb{B}$ or else,

(b) there exist $u \in \mathrm{bd} \mathbb{B}$ and $t>0$ such that $K=L+$ tu and for all $l \in \operatorname{bd} L$ we have $\left(l+T_{u} \mathbb{B}\right) \cap L=\{l\}$ or $\left(l+T_{-u} \mathbb{B}\right) \cap L=\{l\}$.

Proof Without a loss of generality we may assume that $\varrho_{H}^{\mathbb{B}}(K, L)=2$.

Let $K \subset L$ or $L \subset K$. It is easy to see that this assumption implies $K=L+\lambda \mathbb{B}$ or $L=K+\lambda \mathbb{B}$ for some $\lambda>0$ (compare Schneider 1981). 
Let now $K \not \subset L$ and $L \not \subset K$. Let $C:=\frac{1}{2}(K+L)$. If int $C=\emptyset$ then, by Corollary 5.4, there exists $u \in \operatorname{bd} \mathbb{B}$ such that $K=C+u=L+2 u$. Lemma 5.3 implies that $u \dashv$ aff $C$ and for all $d \in \operatorname{aff} C$ we have $\left(d+T_{u} \mathbb{B}\right) \cap$ aff $C=\{d\}$ or $\left(d+T_{-u} \mathbb{B}\right) \cap$ aff $C=\{d\}$.

Let now int $C \neq \emptyset$. By Lemma 5.7, $\operatorname{bd}(K * L) \subset C+\mathbb{R} \cdot u$ and the set $K * L$ does not contain straight lines which are not parallel to $u$. In fact, $(K * L)=C+\mathbb{R} \cdot u$. Let $c \in\left(\operatorname{bd}_{K, L} C \cap \operatorname{bd}_{L, K} C\right)$. Then $c$ belongs to some hyperplane $H$ supporting $K$ and $L$.

If $H \cap C+u=H \cap L$ take $c_{1} \in\left(\operatorname{bd}_{K, L} C \backslash \operatorname{bd}_{L, K} C\right)$. We have $c_{1}+u \in \operatorname{bd}_{K, L} K$ and $c+u \in \operatorname{bd}_{L, K} L$. Also $K \cap\left(c_{1}+u+T_{-u} \mathbb{B}\right)=\left\{c_{1}+u\right\}$ and $L \cap\left(c+u+T_{-u} \mathbb{B}\right)=\{c+u\}$. The intersection $\left(c_{1}+u+T_{-u} \mathbb{B}\right) \cap\left(c+u+T_{-u} \mathbb{B}\right) \cap(K * L)$ is not empty and the sets $K$ and $L$ are visible from some point in $K * L$, which is impossible. Therefore, $H \cap C+u=H \cap K$ and, in consequence, $\left(\operatorname{bd}_{K, L} C \cap \operatorname{bd}_{L, K} C\right)+u \subset \operatorname{bd} K$.

By the last inclusion and Lemma 5.7 we obtain that bd $C+u=\operatorname{bd} K$. Since $C$ and $K$ are convex, $C+u=K$. Moreover, $K=C+u=L+2 u$.

For every $l \in \operatorname{bd}_{K, L} L$ we have $l+2 u \in \operatorname{bd}_{K, L} K$. By Lemma 5.6 (i), $(l+2 u+\mathbb{B}) \cap$ $C=\{l+u\}$. Hence $\left(T_{-u} \mathbb{B}+l+u\right) \cap C=\{l+u\}$. Therefore, $\left(T_{-u} \mathbb{B}+l\right) \cap L=\{l\}$.

Analogously, for every $l \in \operatorname{bd}_{L, K} L$ we have $\left(T_{u} \mathbb{B}+l\right) \cap L=\{l\}$.

\section{Examples}

In this section we show how to construct examples.

Let $u \in \mathbb{R}^{n}$. To simplify the notation, for a given convex body $L$ we shall write $\mathrm{bd}^{*} L$ instead of $\mathrm{bd}_{L, L+u} L$ and $\mathrm{bd}_{*} L$ instead of $\mathrm{bd}_{L+u, L} L$. Certainly, the boundary of $L$ is the union of $\mathrm{bd}^{*} L$ and $\mathrm{bd}_{*} L$. In the following we shall refer to $\mathrm{bd}^{*} L$ and $\operatorname{bd}_{*} L$ as the ceiling of $L$ and the floor of $L$. The set $\mathrm{bd}^{*} L \cap \mathrm{bd}_{*} L$ will be called the seam of $L$.

We can now rephrase Theorem 3.1 as follows.

Theorem 6.1 Let $\mathbb{B}$ be strictly convex and let $K, L \in \mathcal{K}^{n}$. Then the following conditions are equivalent:

(i) the sets $K$ and $L$ have a unique metric segment joining them with respect to the metric $\varrho_{H}^{\mathbb{B}}$;

(ii) either

(a) $K=L+\lambda \mathbb{B}$ or $L=K+\lambda \mathbb{B}$ for some $\lambda>0$

or else,

(b) $K=L+$ tu for $t>0$ and for some $u \in \operatorname{bd} \mathbb{B}$ such that: $\left(T_{u} \mathbb{B}+l\right) \cap L=\{l\}$ and $\left(T_{-u} \mathbb{B}+l\right) \cap L=\{l\}$ for every $l \in \operatorname{bd}^{*} L \cap \operatorname{bd}_{*} L$.

Proof The condition (ii) (b) in Theorem 3.1 can be formulated in a few equivalent ways. The last phrase of this condition can be replaced by: $\left(T_{u} \mathbb{B}+l\right) \cap L=\{l\}$ for every $l \in \operatorname{bd}_{*} L$ and $\left(T_{-u} \mathbb{B}+l\right) \cap L=\{l\}$ for every $l \in \mathrm{bd}^{*} L$. This implies that $\left(T_{u} \mathbb{B}+l\right) \cap L=\{l\}$ and $\left(T_{-u} \mathbb{B}+l\right) \cap L=\{l\}$ for every $l \in \operatorname{bd}^{*} L \cap \operatorname{bd}_{*} L$.

Assume $\mathbb{B}$ to be strictly convex. We construct examples of pairs of convex bodies with a unique metric segment joining them (with respect to the metric $\varrho_{H}^{\mathbb{B}}$ ) as follows: 
Fig. 1 Metric segment joining

$L$ and $L+\lambda \mathbb{B}$
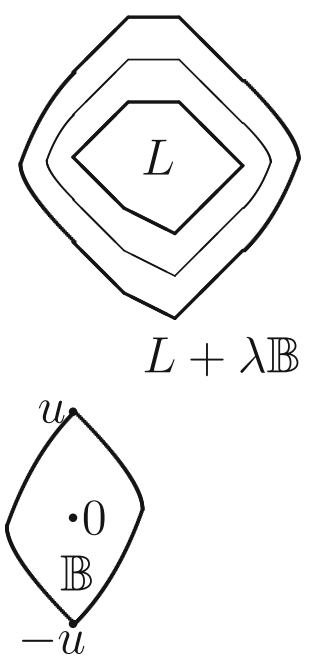

Fig. 2 Metric segment joining $L$ and $L+\lambda u$

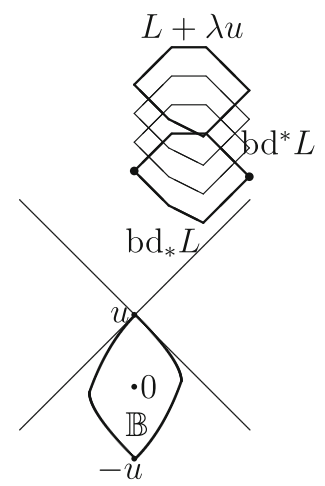

(i) Let $L \in \mathcal{K}^{n}$ and $\lambda>0$. The set $\{L+t \mathbb{B} \mid t \in[0, \lambda]\}$ is the unique metric segment joining $L$ and $L+\lambda \mathbb{B}$ with respect to the metric $\varrho_{H}^{\mathbb{B}}$.

(ii) Let now $L \in \mathcal{K}^{n}, u \in \operatorname{bdB}$ and $\lambda>0$. If for every $l$ from the seam of $L$ the translate of the union of tangent cones $\left(T_{u} \mathbb{B} \cup T_{-u} \mathbb{B}\right)+l$ intersects $L$ in $\{l\}$, then the set $\{L+t u \mid t \in[0, \lambda]\}$ is the unique metric segment joining $L$ and $L+\lambda u$ with respect to the metric $\varrho_{H}^{\mathbb{B}}$.

The following pictures show the situation described in (i) (Fig. 1) and in (ii) (Fig. 2): The following proposition is another consequence of Theorem 3.1.

Corollary 6.2 Let $L \in \mathcal{K}^{n}$ and $u \in$ bdBB. Assume that $L$ and $L+t u$ are endpoints of unique metric segment for some $t>0$. If $T_{u} \mathbb{B}$ contains a straight line, then codim $L \geq 1$ or, equivalently, int $L=\emptyset$.

The following example illustrates this situation:

Let $\mathbb{B}=\left(\mathrm{B}^{n}+x\right) \cap\left(\mathrm{B}^{n}-x\right)$ be a lens. Then $T_{u} \mathbb{B}$ contains a straight line for every $u \in\left(\right.$ bd $\left.\left(\mathrm{B}^{n}+x\right)\right) \cap\left(\mathrm{bd}\left(\mathrm{B}^{n}-x\right)\right)$. Therefore convex bodies $L$ and $L+t u$ are endpoints of unique metric segment for some $t>0$ only if int $L=\emptyset$. 


\section{Conclusions, applications and open problems}

In this paper we have considered Minkowski spaces with strictly convex unit ball. We continue with working on the classification of the unique metric segments in Minkowski spaces with smooth unit ball.

It is also an interesting problem to classify the unique metric segments in Minkowski spaces with arbitrary unit ball in arbitrary dimension.

In the hyperspace $\mathcal{K}^{n}$ we can introduce the notion of extreme element of a subset $\mathcal{X}$ of $\mathcal{K}^{n}$ in a way analogous to that for extreme point of a subset $X$ of $\mathbb{R}^{n}$. Theorem 3.1 enables us to find all extreme elements of balls in the hyperspace $\left(\mathcal{K}^{n}, \varrho_{H}^{\mathbb{B}}\right)$

$$
\mathcal{B}(A, r):=\left\{X \in \mathcal{K}^{n} \mid \varrho_{H}^{\mathbb{B}}(A, X) \leq r\right\}
$$

for $A \in \mathcal{K}^{n}$ and $r>0$.

Proposition 7.1 Let $L \in \mathcal{K}^{n}$ and $r>0$. Extreme elements of the ball $\mathcal{B}(L, r)$ are the following:

(i) $L+r \mathbb{B}$;

(ii) $A \in \mathcal{K}^{n}$ such that $L=A+r \mathbb{B}$ under the condition that $r \mathbb{B}$ is a summand of $L$;

(iii) $L+r u$ for $u \in$ bdB provided that the condition (ii) (b) of Theorem 3.1 is satisfied.

Our result can be formulated in terms of convex functions and subdifferentials. Let $V \subset \mathbb{R}^{n}$ be an $(n-1)$-dimensional subspace not containing $u \in$ bdB. Then $\mathbb{R}^{n}$ is a direct sum of $V$ and $\mathbb{R} \cdot u$. Let $f: \mathbb{R}^{n} \longrightarrow \mathbb{R}$ be a linear function such that $f^{-1}(0)=V$ and $f(u)=1$. Since $u \in \operatorname{int} T_{-u} \mathbb{B}$ and $T_{-u} \mathbb{B}$ is a convex cone, the closed cone $\mathrm{cl} T_{-u} \mathbb{B}$ is an epigraph of some sublinear function $p: V \longrightarrow \mathbb{R}$, that is, bd $T_{-u} \mathbb{B}=\{v+p(v) u \mid v \in V\}$.

Let $L \in \mathcal{K}^{n}$ and $g: V \longrightarrow \mathbb{R} \cup\{\infty\}$ be a function defined by $g(v)=\inf \{t \in$ $\mathbb{R} \mid v+t u \in L\}$. Since $L$ is convex, the function $g$ is also convex.

The following proposition is an obvious corollary of Theorem 5.8.

Proposition 7.2 Let $L \in \mathcal{K}^{n}, u \in \mathrm{bdB}, \lambda>0$, and let $L, L+\lambda u$ be endpoints of a unique metric segment. Then

(i) $g(x)-g(y) \leq f(x-y)$ for all $x, y \in \operatorname{dom} g$,

(ii) $\left.\left.\partial g\right|_{x} \subset \partial f\right|_{0}$ for all $x \in$ int dom $g$.

Acknowledgments The authors wish to thank Maria Moszyńska for careful reading, corrections and valuable suggestions for improvement

Open Access This article is distributed under the terms of the Creative Commons Attribution License which permits any use, distribution, and reproduction in any medium, provided the original author(s) and the source are credited.

\section{References}

Alonso, J., Martini, H., Senlin Wu: On Birkhoff orthogonality and isosceles orthogonality in normed linear spaces. Aequationes Math. 83, 153-189 (1953) 
Blumenthal, L.M.: Theory and applicationes of distance geometry, 1st ed., Clarendon Press, Oxford (1953)

Blumenthal, L.M.: Theory and applicationes of distance geometry, 2nd ed. Chelsea Publ. Co., New York (1970)

Bogdewicz, A.: Some metric properties of hyperspaces. Demonstratio Math. 33, 135-149 (2000)

Bogdewicz, A., Grzybowski, J.: Pairs of convex bodies in a hyperspace over a Minkowski two-dimensional space joined by a unique metric segment, vol. 84, pp. 75-88. Banach Center Publ., Warsaw (2009)

Boltyanski, V., Martini, H., Soltan, P.: Excursions into Combinatorial Geometry. Springer, Berlin/Heidelberg (1997)

Jongmans, F.: De l'art d'être a bonne distance des ensambles dont la décomposition atteint un stade avancé. Bull. Soc. Roy. Sci. Liège 48, 237-261 (1979)

Martini, H., Swanepoel, K.J.: The geometry of Minkowski spaces-a survey, Part II. Expositiones Math. 22, 93-144 (2004)

Menger, K.: Untersuchungen über allgemeine Metrik, I, II, III. Math. Ann. 100, 75-163 (1928)

Schneider, R.: Pairs of convex bodies with unique joining metric segments. Bull. Soc. Roy. Sci. Liège 50, 5-7 (1981)

Schneider R.: Convex Bodies: the Brunn-Minkowski Theory. Cambridge University Press, Cambridge (1993)

Thompson A.C.: Minkowski Geometry. Cambridge University Press, New York (1996) 